\title{
Four years on, clinical partnerships program proves worth
}

Last fall, as the H1N1 'swine flu' pandemic reached full swing, Gordon Bernard of Vanderbilt University Medical Center in Nashville, Tennessee started gearing up to test his idea that statins, which can dampen inflammation, might combat the disease. Whereas clinical trials often take years to get up and running, Bernard's study was approved in record time-two months.

Bernard credits his success to a program that helped him round up research partners and supported him in designing the trial-the Clinical and Translational Science Awards (CTSA) program. The program, launched in 2006 by the US National Institutes of Health (NIH), aims to speed up the process of transforming laboratory discoveries into therapeutic treatments by restructuring the translational research enterprise in the US.

Before the award program, anyone who wanted to conduct human studies faced a gauntlet of 18 institutional bodies at Vanderbilt, says Bernard, who directs the CTSA-funded Vanderbilt Institute for Clinical and Translational Research. Now, many of these groups report to one office. "The CTSA program has transformed the landscape" of clinical research, he says.

Four years on from the CTSA's inception, the NIH has given awards to 46 academic centers with the goal of spending $\$ 500$ million annually to fund a total of 60 institutions by 2012. And this month, all 12 universities and hospitals that received the first CTSA grants are submitting their grant proposals for a fiveyear renewal.

It's difficult to quantify the success of the CTSA program directly because it's mainly designed to promote collaboration and provide support and infrastructure, rather than fund large projects. But many CTSA investigators boast that the program has hammered at the barriers to clinical studies, cutting into red tape, centralizing regulatory offices, training researchers and forging numerous new collaborations, programs and tools.

In the Boston region, five major universities and hospitals came together under the Harvard 'Catalyst' center, and in the Northwest, seven institutions in Seattle and community health organizations across five states banded together to form the CTSA-funded Institute of Translational Health Sciences. In Philadelphia, meanwhile, $\$ 65$ million in CTSA funding spurred the expansion of the Institute for Translational Medicine and Therapeutics, through an alliance among its supporting institutions, including the University of Pennsylvania and Children's

\section{Tool kit for translational research}

The CTSA program has sped up the pace of research by promoting shared informatics tools, such as the four featured here. Not all of these tools are funded fully by the CTSA — but the program has enabled individual institutions to support these projects and fostered their dissemination.

\section{Project: REDCap (Research Electronic Data Capture)}

In 2004, when Paul Harris, a biomedical informaticist at Vanderbilt University in Nashville, Tennessee, began to create software for managing research data, the process of collecting results for biomedical studies was a mess. Many researchers relied on Excel spreadsheets, and if the person managing the data left the lab, it was often hard to decipher the results. Worryingly, many systems also didn't comply with new nationwide privacy rules pertaining to research involving human subjects.

So Harris, who directs Vanderbilt's CTSA Biomedical Informatics Operations, and his team created REDCap, a

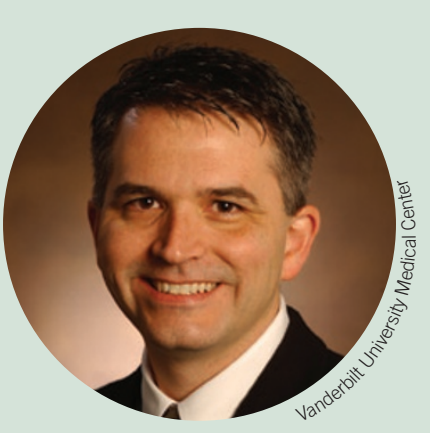
web-based application for designing tailor-made systems for entering and retrieving study data. Because researchers can access the platform remotely, the system makes it easy to manage studies occurring at multiple clinical sites around the world, notes Harris,

Since expanding beyond Vanderbilt in 2006, REDCap has caught on at 95 institutions worldwide, many of them CTSA-funded, and supports 1,700 studies. Users provide feedback to Harris' team so they can continuously improve the platform. "With this, you can be a collaborator without even knowing what the word CTSA means," Harris says.

Website: https://redcap.vanderbilt.edu/

\section{Project: VIVO}

Mike Conlon does not like his project to be compared to Facebook. "That is a description written by a journalist for my mom," says Conlon, an informatics researcher at the University of Florida-Gainesville who is in charge of VIVO, an ambitious social networking project for scientists. Like Facebook, VIVO provides profiles of scientists that look much like any departmental web page. But unlike Facebook, the data are in a standardized format that allows precise searches and enables developers to whip up a lot of 'apps'.

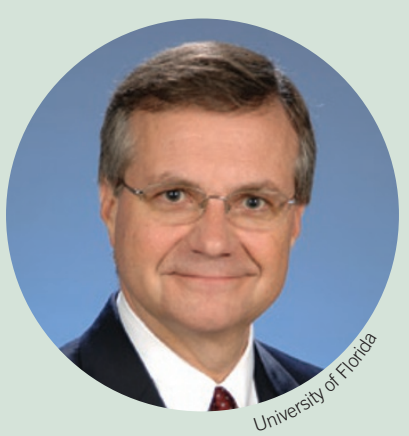

VIVO links up with databases such as PubMed to automatically collect updated information on publications and grants, which allows use of the open-source tool to visually display author collaborations or automatically compile directories showcasing various scientists' expertise. "We are thinking about how to hook everything up," says Conlon, who collaborates with VIVO's original developer, Jonathan Corson-Rikert at Cornell University in Ithaca, New York.

Last year, Conlon snagged a $\$ 12.2$ million 'stimulus' grant to disseminate VIVO, which is already being deployed at Indiana University in Bloomington, Washington University in St. Louis and five other institutions. But whether VIVO catches on with scientists remains to be seen, cautions Nick Anderson, associate director of bioinformatics at the University of Washington's CTSA-funded institute in Seattle. Other online tools could rival VIVO, and most collaborations are still forged face to face, he says. "It's done over beer."

Website: http://www.vivoweb.org/ 


\section{Project: ResearchMatch}

In 2008, Robert Hoffman set out to test the idea that differences in how blood vessels respond to insulin can explain racial differences in disease risk. The only problem: he couldn't find enough subjects willing to enroll in his study.

Hoffman, who studies diabetes and blood pressure at Ohio State University in Columbus, needed ten individuals of European descent and ten African-American volunteers willing to stay overnight at a local research center and be pricked with a few needles. He had advertised in the campus newspaper and distributed flyers, but after searching for 18 months and spending $\$ 2,000$ he still had found only two black participants. "I was really having a hard time recruiting," he recalls.

Then, in April, he learned about ResearchMatch, a nationwide registry of more than 7,000 — and counting — volunteers willing to participate in research studies. Hoffman e-mailed dozens of the anonymous participants who fit the profile he needed, and days later he had four additional African-American enrollees.

ResearchMatch, developed by Paul Harris and colleagues at Vanderbilt University, went live this March at 41 CTSA institutions, and more than 550 volunteers have already been directly connected with researchers through the system.

The registry promises to speed up enrollment of research subjects, a lengthy process that has gummed up clinical trials nationwide, says Rose Hallarn, who directs clinical trial recruitment at the Ohio State's CTSA program. "You could do your entire recruitment through ResearchMatch," she says.

Website: https://www.researchmatch.org/

\section{Project: i2b2 (Informatics for Integrating Biology and the Bedside)}

Isaac Kohane has a vision. He imagines a nationwide effort to collect data on millions of patients at hospitals and clinics dotted throughout the country. Such data-collected with consent, and used in anonymous databases-could help researchers spot adverse drug effects and find people to enroll in clinical trials, among other uses. Kohane and his colleagues have made the tool that they think can make that vision become reality: $\mathrm{i} 2 \mathrm{~b} 2$.

$12 \mathrm{~b} 2$ is an open-source platform now used by

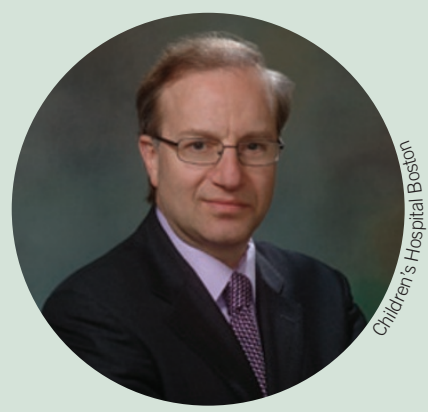
more than 40 academic health centers that enables researchers to collect patient information from electronic health records and other sources. The information can be shared across systems by authorized individuals using peer-to-peer networks-"much like old file-sharing systems gathered mp3 music files," says Kohane, a pediatrician and bioinformatics researcher who co-directs the Harvard Medical School Center for Biomedical Informatics in Boston.

With i2b2, Kohane's team gathered patient data from two different institutions within the Boston-area CTSA. The data revealed associations between heart attacks and the drugs Vioxx (PLoS One 2, e840, 2007) and Avandia (Diabetes Care 3, 526531,2010 )-links that had previously only emerged after years of expensive clinical studies. A Harvard team led by geneticist Robert Plenge is also deploying i2b2 in a genome-wide association study of rheumatoid arthritis by analyzing DNA and blood samples from people unknown to the researchers. - CS

Website: https://www.i2b2.org/
Hospital of Philadelphia. "Traditionally, institutions compete with a verve that inversely correlates with the distance that separates them," says Penn's Garret FitzGerald, who heads the five-year award, initiated in 2006. "This was a big deal."

FitzGerald's experience is echoed down the line by other CTSA leaders. "Competitors are now suddenly really engaged in working together," says Anantha Shekhar, director of the Indiana Clinical and Translational Sciences Institute, which includes Indiana University, Purdue University and the University of Notre Dame, among others.

\section{Lost in translation}

Despite its successes, the program has been criticized for not addressing the earliest stages of the therapeutic pipeline. One main reason, many say, is that the CTSA has not had the power to change the academic reward system for bench researchers. "In academia, how do you develop the incentive for someone to do something?" asks Caren Heller, assistant dean for intercampus initiatives at Weill Cornell Medical College in New York who analyzed the 12 initial CTSA programs last year (Acad. Med. 84, 424-432, 2009). "You either give money or you promote them academically, through tenure and such. And the CTSA can't do either."

FitzGerald points out that basic researchers face short grant cycles and come up for tenure quickly, both factors incompatible with the time involved in moving a potential therapy into a human study. Nevertheless, he sees that change is coming. "I think from the very beginning we knew we were undertaking an attempt at culture change," FitzGerald says. "This is an extended issue, not a one- or two- or five-year project."

Such institutional change should also include fostering links with industry and counting achievements such as number of commercial licenses, says Lesa Mitchell, vice president of advancing innovation at the Kauffman Foundation in Kansas City, Missouri. "It's time to start harvesting much of the basic research that is now in place," she says. "It takes an entire ecosystem."

Despite such challenges, Bernard says that the CTSA program benefits investigators at all stages of research by shoring up infrastructure and by luring funding from philanthropists and other outside sources.

As for Bernard's own trial of statins, the pandemic fizzled out before he had fully enrolled his subjects. But, like most CTSA heads, Bernard feels he is just getting started with transforming the system. "It's a work in progress," he says. "You don't change institutions and agencies overnight."

Charlotte Schubert, Washington, DC 